\title{
Recurrent lumbar disc herniation
}

\author{
Karin R. Swartz, M.D., AND Gregory R. Trost, M.D. \\ Department of Neurological Surgery, University of Wisconsin Hospital and Clinics, Madison, \\ Wisconsin
}

\begin{abstract}
Recurrent lumbar disc herniation is a common disease process. It has been noted to occur in 5 to $15 \%$ of cases surgically treated for primary lumbar disc herniation. Outcomes in one series approached those after the initial operations, although this is not the case in the experience of most surgeons.

The removal of recurrent lumbar disc herniations requires meticulous surgical technique. Great care is taken to identify the osseous margins of the previous surgical site. Identification and dissection of scar from the dura mater is greatly aided with the use of a microscope.
\end{abstract}

KEY WORDS • disc herniation • lumbar spine • recurrence

Single-level lumbar discectomy has been proven to have lasting benefit in numerous cases. Good outcomes depend on proper patient selection; however, complications may arise. Discectomy-related complications occur in 15 to $30 \%$ of cases and include hemorrhage, soft-tissue infection, nerve root injury, dural tear, recurrent or residual disc herniation, epidural scar formation, discitis, arachnoiditis, pseudomeningocele, facet joint fracture (iatrogenic or stress related), spinal stenosis, and epidural hematoma. ${ }^{2,17,19,21,24}$ Potential predictors of poor outcome include misdiagnosis (for example, diabetic polyneuropathy mistaken for radiculopathy), preoperative psychological distress, insufficient rehabilitation, mechanical instability, impaired fibrinolytic activity, diabetes, obesity, and hypertension. ${ }^{1,5,14,16,17}$

The rate of recurrent disc herniation after lumbar discectomy is 5 to $15 \%$. $^{2,7,17,20,24}$ The strict definition of recurrent disc herniation is the presence of herniated disc material at the same level, ipsi- or contralateral, in a patient who has experienced a pain-free interval of at least 6 months since surgery. The clinically more appropriate definition, however, is disc herniation at the previously operative site and side. The pain-free interval should not be restricted to the minimum of 6 months. It has been suggested that the mean interval for recurrent pain associated with recurrent herniated discs is 18 months, longer than that for de novo herniated discs or symptomatic epidural fibrosis. ${ }^{10}$

Treatment options of first-time disc herniations include observation combined with aggressive medical manage-

Abbreviation used in this paper: $\mathrm{MR}=$ magnetic resonance. ment (pharmacological and physical therapies), chymopapain, intradiscal electrothermal coagulation therapy, laserassisted decompression, laminectomy, laminectomy and discectomy, minimally invasive microdiscectomy and endoscopic discectomy, and laparoscopic discectomy; surgical choices for disc recurrent herniations are limited by multiple factors, require longer operative time, and are associated with higher rate of complications, treatment seems to be associated with a similar chance of good outcome. . $^{8,10,13,23,24}$

\section{RECURRENT LUMBAR DISC HERNIATIONS}

\section{Risk Factors}

There are numerous risk factors for recurrent disc herniation. In patients with diabetes, hospitalization is prolonged and there is a higher risk of postoperative infection as well as poorer long-term results. Simpson, et al. ${ }^{22}$ reported excellent/good outcomes in 39\% of their patients with diabetes and $95 \%$ of those without diabetes, in cases in which an initial discectomy was performed. In their retrospective analysis, Mobbs, et al.,17 noted a slightly higher rate of excellent/good outcome; whereas it was $60 \%$ in their diabetic group, it remained significantly lower (86\%) in the nondiabetic group. This may be attributable to lower quality of life indicators in diabetic compared with nondiabetic individuals. Robinson, et al., ${ }^{18}$ analyzed the proteoglycan profile in the intervertebral discs of diabetic and nondiabetic patients, and determined that there were fewer proteoglycans in the former group, which may increase susceptibility to disc prolapse in patients with diabetes. 
One controversial risk factor for recurrence is the shape of the disc itself. Grane, et al., ${ }^{13}$ and Suk, et al.,${ }^{24}$ have asserted that disc shape plays no part in recurrence. Carragee, et al., ${ }^{7}$ prospectively evaluated disc herniation types, rate of reherniation, and rate of reoperation. They divided disc herniations into four shape-based groups: 1) fragment-fissure herniations (disc fragment and small anular defect); 2) fragment-defect herniations (large disc fragment with massive posterior anular tear); 3) fragmentcontained discs (incomplete anular tear); and 4) absence of fragment-contained herniations (anular prolapse). Of the four groups, the fragment-fissure type herniations were associated with the best outcomes, lowest rate of reherniation $(1 \%)$, and required the fewest reoperative procedures $(1 \%)$. Those with anular prolapse were associated with the worst outcomes, with $38 \%$ of patients experiencing recurrent or persistent symptoms.

Although work type and profession were not found to be risk factors, patients filing Workers' Compensation claims experienced poorer outcomes than those not making claims. ${ }^{7}$ Sex, age, smoking status, level of herniation, and duration of symptoms were not associated with higher rates of recurrence. ${ }^{7,8,14,17,24}$

\section{Minimizing Risks of Recurrence}

In an attempt to minimize postoperative complications in general, early postoperative mobilization of patients is strongly recommended. Many proposed risk factors have not been substantiated - neither the act of anular incision nor extent of discectomy, for example, has been shown to influence recurrence. . $^{8,17,17}$ Not only higher recurrence rates but also poorer outcomes have been documented in patients with diabetes; ${ }^{17}$ it has not yet been shown if close control of diabetes has any effect.

\section{Evaluation of Recurrence}

Many modalities have been used to evaluate the lumbar spine after surgery. The current neuroimaging tool of choice is Gd-enhanced MR imaging to investigate postdiscecetomy recurrent symptoms. ${ }^{2}$ Variations, including fat saturation, $\mathrm{T}_{2}$-weighted turbo-spin echo, turbo fluidalternated inversion recovery, short-tall inversion recovery, and differing contrast media have been suggested to increase sensitivity and specificity of MR imaging., ${ }^{2,311,15}$ Most practitioners agree that optimal evaluation requires that the patient undergo neuroimaging immediately after contrast administration because the enhancement pattern is best within the first 5 minutes; beyond that, enhancement in the disc can be seen if imaging is delayed (Figs. 1 and 2). ${ }^{2}$

To recognize postoperative pathological changes, one must be familiar with changes appropriate to early and late phases. Normal postdiscectomy appearances can be mistaken for recurrent or retained disc. In the early ( 0 to 6-month) postoperative period, MR imaging reveals an interspace high signal intensity band extending from the nucleus pulposus to the site of anular disruption (especially noticeable at $0-2$ months). The anulus is typically hyperintense and the nucleus hypointense. There is loss of disc space height. The endplates and marrow can exhibit changes as well, often low signal on $\mathrm{T}_{1}$-weighted and high signal on $\mathrm{T}_{2}$-weighted images suggesting inflammation and edema. The anterior epidural space initially reveals an increase in soft-tissue mass, evidence of tissue disruption, edema, and hemorrhage, with the appearance of mass effect. ${ }^{20}$ Nerve root enhancement with Gd is normal, reflecting breakdown of the blood-nerve barrier, but should resolve by 6 months. Adhesions within the thecal sac at the operative level usually resolve within several weeks. Postoperative changes at the laminectomy site depend on the extent of surgery, ligamentum flavum removal, and whether fat graft was placed in the epidural space. Facet joint enhancement occurs as a local response to dissection and persists long ( $>6$ months) after surgery in more than half of the patients in whom imaging is performed., 2,6,25

Normal late ( $>6$ months) postdiscectomy changes are those of healing. The now low-intensity signal band in the disc space represents a healing anular defect. The mass effect seen earlier in the anterior epidural space may have resolved ${ }^{25}$ or may persist as a masslike scar. ${ }^{9}$ Scarring is most pronounced before 9 months ${ }^{12}$ and primarily involves the anulus fibrosus. The nerve root should not enhance after 6 months. ${ }^{6}$ The laminectomy space exhibits matured scar with peripheral enhancement identifying granulation tissue. Facet joint enhancement is visible after contrast administration in approximately half of the patients 6 months postoperatively. ${ }^{2}$

Pathological changes of the anterior epidural space can reflect mass effect due to scarring or the disc. ${ }^{2,9}$ Differentiation between these is central in choosing the best treatment option because the former will not benefit from surgery but the latter may. Both are demonstrated to have similar signal intensities on unenhanced $\mathrm{T}_{1}$-weighted $\mathrm{MR}$ images. ${ }^{15}$ Scarring often displays intermediate signal intensity; beyond 2 years, it may be hypointense. Scarring enhances heterogeneously because of its vascular supply. The disc usually appears as a polypoid mass of low signal on $\mathrm{T}_{1}$ - and $\mathrm{T}_{2}$-weighted sequences. It is usually contiguous with the parent disc unless sequestrated. There is often a hypointense rim of the posterior longitudinal ligament and outer anular fibers outlining the herniation, enhancing after contrast administration. The disc itself does not enhance because it has no blood supply. ${ }^{2,15}$ Retraction of the thecal sac toward a soft-tissue lesion is suggestive of scar ${ }^{20}$ displacement away from such a mass is suggestive of a herniated disc. Although a pseudomeningocele may also be seen as mass, its signal characteristics are different, identifying them as such: cerebrospinal fluid intensity on $\mathrm{T}_{1}$ - and $\mathrm{T}_{2}$-weighted images and often an enhancing fibrous capsule. ${ }^{2}$

What constitutes identifiable differences between symptomatic and asymptomatic postoperative MR imaging changes is not well understood; a mass lesion at the level of previous surgery is a common finding, especially in the early postoperative period. ${ }^{13}$ Recurrent pain prompting postoperative neuroimaging is poorly specific in nature and is difficult to interpret. ${ }^{21}$ Discordance between neuroimaging and intraoperative findings is more common than anticipated, occurring in 18 to $33 \%$ of the cases proven surgically. ${ }^{10}$

\section{Treatment of Recurrence}

Differentiation of recurrent disc hemiation from scar formation will allow for improved treatment choices and 


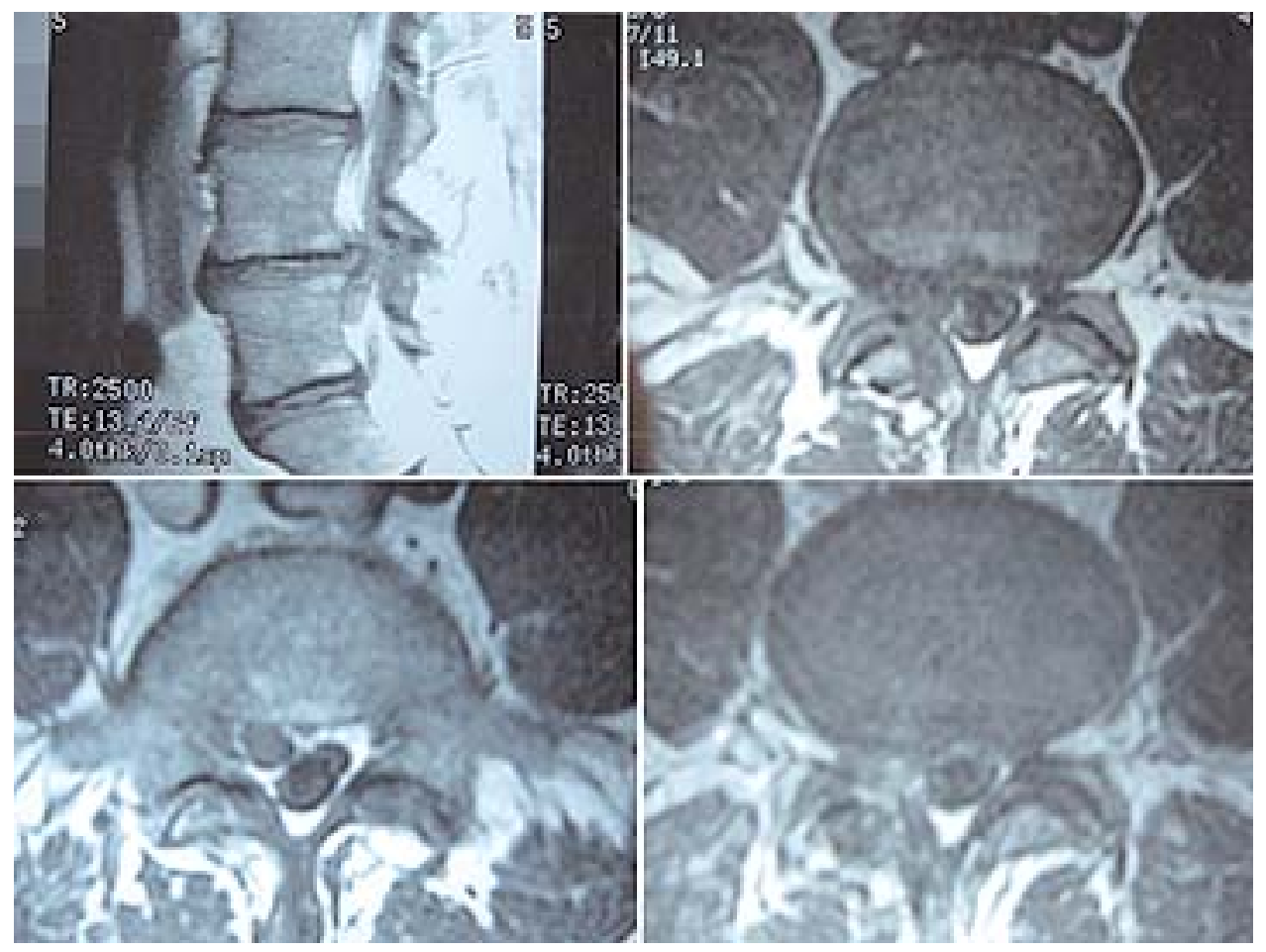

Fig. 1. Representative MR images. Upper Left: Sagittal $\mathrm{T}_{1}$-weighted image demonstrating recurrent L4-5 disc herniation. Upper Right: Axial $\mathrm{T}_{1}$-weighted image without contrast of recurrent disc herniation. It is difficult to differentiate between scar and disc fragment in this image. Lower Left: Image revealing recurrent disc herniation with caudal migration. Similarly there is no differentiation between scar, nerve root, and disc herniation. Lower Right: Contrastenhanced image demonstrating lack of enhancement of soft-tissue suggestive of recurrent disc herniation.

selection of patients who may benefit from a second surgery. Gadolinium-enhanced MR imaging is thought to be the best modality to differentiate between the two. ${ }^{2,3,11,20,25}$ Computerized tomography discography may also provide additional information. ${ }^{4}$ The scar may surround the nerve roots and cause symptoms by means of neural tension, decreased axoplasmic transport, restriction of blood flow, or of venous return. ${ }^{2}$ In classical teaching, a scar does not benefit from reoperation and in fact may result in worse outcomes. ${ }^{13}$

Treatment options include observation and aggressive medical management (pharmacological therapy and physical therapy for rehabilitation) or operative intervention. Revision laminectomy and discectomy are the most commonly performed surgical therapies, starting at an area known to be intact, finding landmarks, beginning medially, and working out laterally to locate the pathological entity. In creating the surgical exposure, the surgeon should concentrate on removing scar from the lamina to make a clear identification of the previous laminotomy edges. Curettes are then used to dissect the scar from the osseous margins. Care must taken to delineate meticulously the bone from scar to avoid violating the dura mater. It is generally easier to dissect laterally in the canal and work medially. Identification of pedicles allows for clean separation of the scar tissue from bone as well as identification of the disc space. The use of an operative microscope greatly improves identification and separation of tissues. Chymopapain, intradiscal electrothermal coagulation therapy, and laser-assisted decompression are not options because the anulus is no longer intact in revision disc surgery. Fusion is not routinely needed, unless spinal instability is demonstrated.

In an analysis of recurrent herniated disc surgery in the setting of conventional open discectomy, Suk, et al., ${ }^{24}$ found no significant differences in factors influencing the outcomes of repeated discectomy, in terms of sex, age, smoking status, trauma status, degree of degenerative disease, and whether the anulus was incised during surgery. Cinotti, et al., ${ }^{8}$ reported no difference in recurrence rates when comparing cases of partial and complete discectomy. There is no significant reported effect related to the original surgery and revision surgery on hospital stay and clinical improvement rates; reoperation for recurrent disc herniation has been associated with good outcome, comparable with that achieved after initial surgery. ${ }^{10,13,24}$

\section{CONCLUSIONS}

The precise mechanism by which to explain radicular pain secondary to lumbar disc disease, both initial and recurrent, is not fully understood. Implicated are direct mechanical pressure, vascular changes, and inflammatory stimuli due to herniated material. ${ }^{17}$ Higher recurrence rates and poorer outcomes have been documented in diabetic patients, findings that accentuate the importance of patient education and preparation. ${ }^{17,18,22}$ Whether open laminectomy combined with discectomy, microdiscecto- 


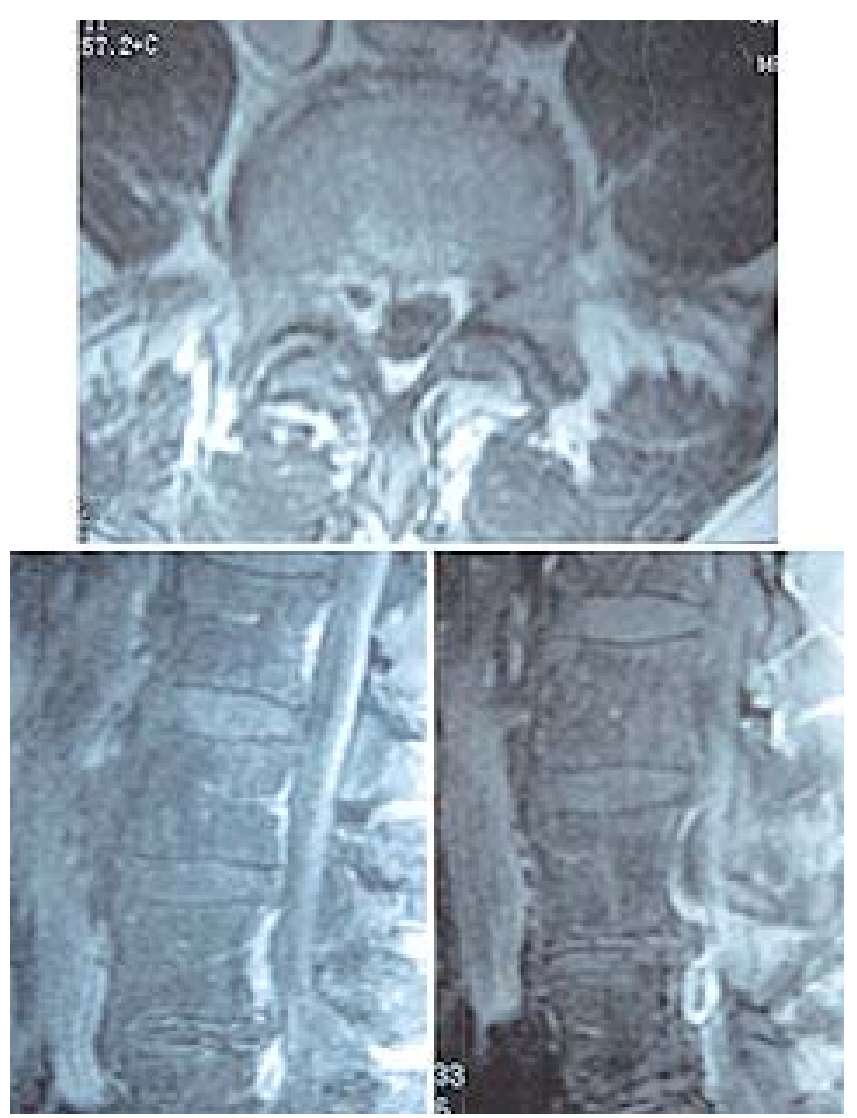

Fig. 2. Representative MR images. Upper: Contrast-enhanced image demonstrating an extruded fragment. Lower Left: Lack of enhancement of material in continuity with the disc space strongly suggests a disc herniation. Contrast enhancement can be seen adjacent to the disc material. Lower Right: Contrast (scar) surrounds the extruded fragment of disc.

my, minimally invasive endoscopic discectomy, or laparoscopic discectomy will yield divergent recurrence rates has not been fully elucidated, but preliminary comparisons have yet to reveal significant differences. ${ }^{17,23}$ Likewise, there is no consensus on timing or optimal intervention in the treatment of patients with recurrent herniated discs.

\section{References}

1. Airaksinen O, Herno A, Turunen V, et al: Surgical outcome of 438 patients treated surgically for lumbar spinal stenosis. Spine 22:2278-2282, 1997

2. Babar S, Saifuddin A: MRI of the post-discectomy lumbar spine. Clin Radiol 57:969-981, 2002

3. Barrera MC, Alustiza JM, Gervas C, et al: Post-operative lumbar spine: comparative study of TSE T2 and turbo-FLAIR sequences vs contrast-enhanced SE T1. Clin Radiol 56:133-137, 2001

4. Bernard TN Jr: Using computed tomography/discography and enhanced magnetic resonance imaging to distinguish between scar tissue and recurrent lumbar disc herniation. Spine 19: 2826-2832, 1994

5. Bernsmann K, Krämer J, Ziozios I, et al: Lumbar micro disc surgery with and without autologous fat graft. A prospective randomized trial evaluated with reference to clinical and social factors. Arch Orthop Trauma Surg 121:476-480, 2001
6. Boden SD, Davis DO, Dina TS, et al: Contrast-enhanced MR imaging performed after successful lumbar disk surgery: prospective study. Radiology 182:59-64, 1992

7. Carragee EJ, Han MY, Suen PW, et al: Clinical outcomes after lumbar discectomy for sciatica: the effects of fragment type and anular competence. J Bone Joint Surg Am 85:102-108, 2003

8. Cinotti G, Roysam GS, Eisenstein SM, et al: Ipsilateral recurrent lumbar disc herniation. A prospective, controlled study. J Bone Joint Surg Br 80:825-832, 1998

9. Deutsch AL, Howard M, Dawson EG, et al: Lumbar spine following successful surgical discectomy. Magnetic resonance imaging features and implications. Spine 18:1054-1060, 1993

10. Erbayraktar S, Acar F, Tekinsoy B, et al: Outcome analysis of reoperations after lumbar discectomies: a report of 22 patients. Kobe J Med Sci 48:33-41, 2002

11. Georgy BA, Hesselink JR, Middleton MS: Fat-suppression contrast-enhanced MRI in the failed back surgery syndrome: a prospective study. Neuroradiology 37:51-57, 1995

12. Glickstein MF, Sussman SK: Time-dependent scar enhancement in magnetic resonance imaging of the postoperative lumbar spine. Skeletal Radiol 20:333-337, 1991

13. Grane P, Tullberg T, Rydberg J, et al: Postoperative lumbar MR imaging with contrast enhancement. Comparison between symptomatic and asymptomatic patients. Acta Radiol 37: 366-372, 1996

14. Graver V, Haaland AK, Magnaes B, et al: Seven-year clinical follow-up after lumbar disc surgery: results and predictors of outcome. Br J Neurosurg 13:178-184, 1999

15. Haughton V, Schreibman K, De Smet A: Contrast between scar and recurrent herniated disk on contrast-enhanced MR images. AJNR 23:1652-1656, 2002

16. Kardaun JW, While LR, Shaffer WO: Acute complications in patients with surgical treatment of lumbar herniated disc. J Spinal Disord 3:30-38, 1990

17. Mobbs RJ, Newcombe RL, Chandran KN: Lumbar discectomy and the diabetic patient: incidence and outcome. J Clin Neurosci 8:10-13, 2001

18. Robinson D, Mirovsky Y, Halperin N, et al: Changes in proteoglycans of intervertebral disc in diabetic patients. A possible cause of increased back pain. Spine 23:849-856, 1998

19. Ross JS: Magnetic resonance imaging of the postoperative spine. Semin Musculoskelet Radiol 4:281-291, 2000

20. Ross JS: MR imaging of the postoperative lumbar spine. Magn Reson Imaging Clin N Am 7:513-524, 1999

21. Sarrazin JL: [Imaging of postoperative lumbar spine.] J Radiol 84:241-250, 2003 (Fr)

22. Simpson JM, Silveri CP, Balderston RA, et al: The results of operations on the lumbar spine in patients who have diabetes mellitus. J Bone Joint Surg Am 75:1823-1829, 1993

23. Slotman GJ, Stein SC: Laminectomy compared with laparoscopic diskectomy and outpatient laparoscopic diskectomy for herniated L5-S1 intervertebral disks. J Laparoendosc Adv Surg Tech A 8:261-267, 1998

24. Suk KS, Lee HM, Moon SH, et al: Recurrent lumbar disc herniation: results of operative management. Spine 26:672-676, 2001

25. Van de Kelft EJ, van Goethem JW, de La Porte C, et al: Early postoperative gadolinium-DTPA-enhanced MR imaging after successful lumbar discectomy. Br J Neurosurg 10:41-49, 1996

Manuscript received July 28, 2003.

Accepted in final form August 19, 2003.

Address reprint requests to: Gregory R. Trost, M.D., Department of Neurological Surgery, University of Wisconsin Hospital and Clinics, 600 Highland Avenue, Madison, Wisconsin 53792. email: trost@ neurosurg.wisc.edu. 